\title{
Epidemiology of uveitis in the mid-Atlantic United States
}

This article was published in the following Dove Press journal:

Clinical Ophthalmology

20 May 2015

Number of times this article has been viewed
Purpose: To demonstrate the demographic, anatomic, and diagnostic classification of patients with uveitis seen in a tertiary care center in central Virginia.

Methods: Retrospective chart review of patient demographics, disease characteristics, and disease severity-related outcomes (therapies, visual outcomes, and complications) from 1984 to 2014 .

Results: There were 491 patients ( 644 eyes) with mean age of 46 years ( \pm 21.4 years) and mean duration of follow up of 4.8 years ( \pm 6.8 years). Of these, 278 patients were female $(56.6 \%)$. Further, $60.5 \%$ were Caucasian, and $27.3 \%$ were African American. The anatomic types seen were anterior uveitis $(67.3 \%)$, panuveitis $(14.5 \%)$, posterior uveitis $(12.6 \%)$, and intermediate uveitis $(5.3 \%)$. The most common etiology was post-traumatic (12.2\%), followed by post-procedural (10.0\%), herpetic (7.9\%), human leukocyte antigen (HLA)-B27-associated (6.7\%), and sarcoidosis (6.7\%). Herpetic uveitis was more common among Caucasians than African Americans (sex-adjusted odds ratio [OR]: 7.69, 95\% confidence interval [CI] [2.12, 50.00]), and sarcoidosis was more common among African Americans than Caucasians (sex-adjusted OR: 6.54, $95 \%$ CI $[2.98,15.29])$. Herpetic anterior uveitis was more common among females than males (race-adjusted OR: 3.03 , 95\% CI [1.32, 7.71]). Multifocal choroiditis was more common among males than females (race-adjusted OR: 9.09, 95\% CI $[1.47,100.00])$. Mean logMAR visual acuity was 0.18 at initial and final visit. A total $388(79 \%)$ and $133(27.3 \%)$ patients received local and systemic steroids, respectively. A total 52 patients (10.6\%) received an antimetabolite. A total 116 patients (23.7\%) were managed with topical glaucoma medication. A total 43 (8.8\%), 129 (26.4\%), and 46 patients (9.4\%) underwent glaucoma surgery, cataract surgery, and vitrectomy, respectively.

Conclusion: Over the period of this study, Caucasian patients were more frequently seen than non-Caucasians, although African Americans constituted a considerable size of study population. The most common diagnoses were undifferentiated anterior uveitis, traumatic uveitis, post-procedural uveitis, herpetic disease, HLA-B27 associated uveitis, and sarcoidosis. Unlike previous reports, traumatic and post-procedural uveitis were frequently reported. Mean visual acuity remained stable from initial to final visit.

Keywords: mid-Atlantic United States, central Virginia, demographics

\section{Introduction}

Uveitis is a leading cause of visual morbidity and causes approximately 30,000 new cases of legal blindness annually in the United States alone. ${ }^{1,2}$ Because uveitis encompasses many heterogeneous disorders, factors that vary regionally, such as age, sex, ethnicity, environmental exposures, and genetics, influence ocular inflammation in ways that are incompletely understood..$^{3,4}$

Worldwide, epidemiologic reports on uveitis have led to the identification of new entities, contributed to monitoring of shifting patterns of uveitis, and guided diagnostic and therapeutic approaches. ${ }^{5-12}$ Infectious uveitides, for example, are strongly associated
Correspondence: Ashvini K Reddy Department of Ophthalmology, University of Virginia, I 300 Jefferson Park Avenue, Charlottesville, VA 22908, USA

$\mathrm{Tel}+\mathrm{I} 4342435890$

Fax +l 4349245180

Email ash.vee.knee@gmail.com 


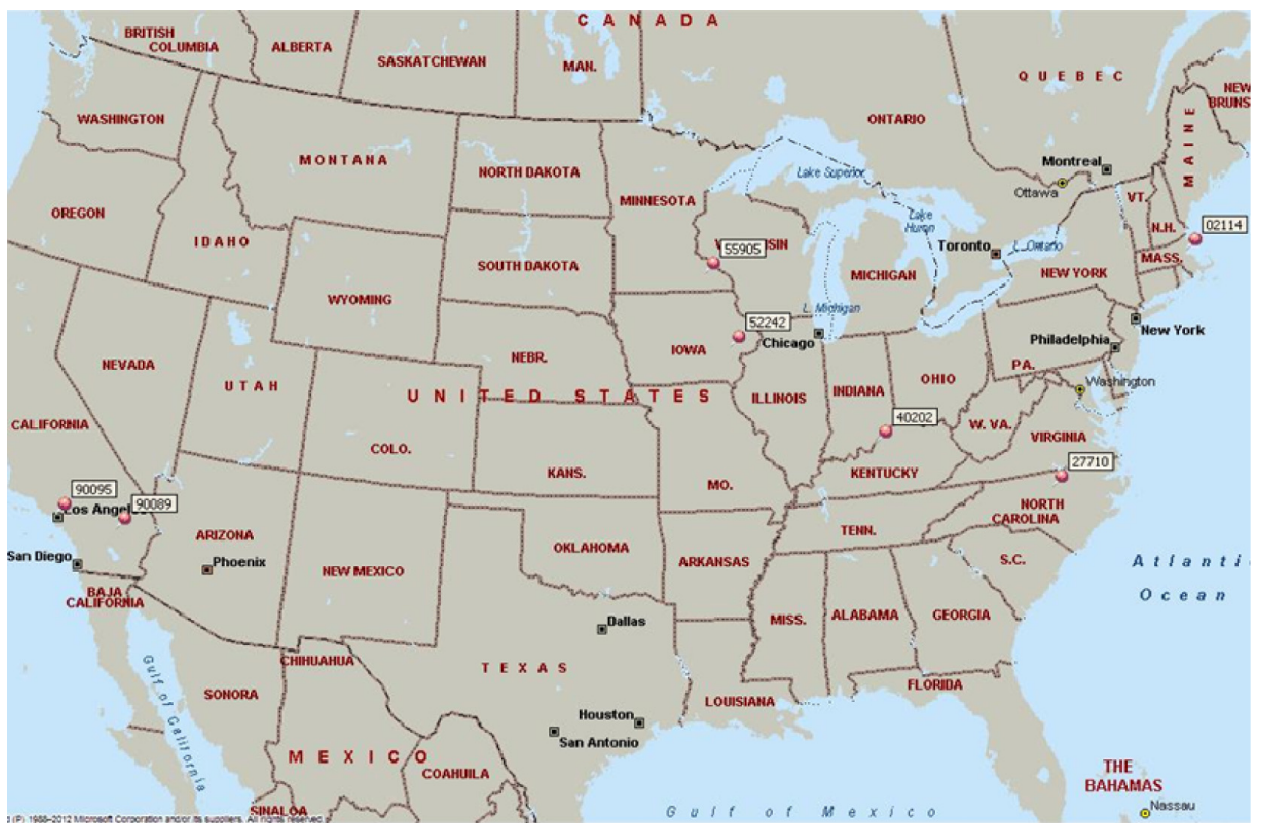

Figure I Map showing centers of the US-based epidemiological studies (created in Microsoft MapPoint ${ }^{\circledR}$ 20I3; Microsoft Corp, Redmond, WA, USA). Note: The red dots indicate center locations.

with geography (ie, toxoplasmosis in Brazil, onchocerciasis in Africa, and histoplasmosis in the Ohio River Valley). ${ }^{6}$ In the United States, there has been increasing interest in population-based studies of uveitis, with growing recognition that demographics and regional factors affect presentation, management, and outcomes (Figure 1). ${ }^{5,-11}$ To date, no such reports exist for the mid-Atlantic United States.

This is a retrospective review of the epidemiologic features of all patients seen with uveitis over a 30-year period at the University of Virginia (UVA), a tertiary referral center for a racially diverse population, attracting patients from Virginia (VA) and neighboring states.

\section{Methods}

Following Institutional Review Board approval, a retrospective review was performed of 1,238 patients seen at UVA from 1984 to 2014 with uveitis identified by 2010 International Classification of Disease, 9th revision (ICD-9) codes corresponding to ocular inflammatory conditions. Individual charts were reviewed, and 747 patients were excluded because a diagnosis of uveitis meeting International Uveitis Study Group criteria could not be confirmed by an attending physician (AKR). ${ }^{13}$ Owing to the long-term nature of this study and reliance on paper records, not all data was available on all patients. To improve our ability to compare our findings with those of nearby centers, patients with scleritis and episcleritis without intraocular inflammation were also excluded. ${ }^{5}$ However, we included cases of cytomegalovirus (CMV) uveitis.
Demographic data, including age, sex, ethnicity, payer status, duration of follow up, number of eye clinic visits, and postal code of residence were recorded. Complete ophthalmic examination findings including best corrected visual acuity, pupillary response, slit-lamp examination, intraocular pressure, and fundoscopic exam were also recorded. Optical coherence tomography, fluorescein angiography, automated perimetry, and other ancillary tests, including serology, radiology, microbiology, and biopsy, were performed when appropriate. Intraocular infection was confirmed with fluid sampling or biopsy, for microscopy, cytology, and culture, or polymerase chain reaction, when appropriate. Post-procedural uveitis was defined as ocular inflammation following intraocular surgery, laser, or intravitreal injection. The term undifferentiated uveitis was applied if intraocular inflammation could not be attributed to a recognized uveitic entity.

Uveitis was classified by anatomic involvement as either anterior, intermediate, posterior, or panuveitis as per Standardized Uveitis Nomenclature (SUN) criteria. ${ }^{14}$ SUN criteria were also used to describe the uveitis as unilateral or bilateral and acute or insidious. Details of management, including the use of local and systemic steroids, antihypertensive drops, intravitreal injections, sub-Tenon injections, antimetabolites, anti-tumor necrosis factor (TNF) agents, cataract extraction, pars plana vitrectomy, and glaucoma management were recorded. Mild visual loss was defined as $>20 / 50(\log M A R<0.4)$ Snellen visual acuity, moderate visual loss as 20/50-20/200 (logMAR 0.4 to $<1.0)$, and severe visual loss as $<20 / 200(\log M A R \geq 1.0)$. 


\section{Statistical analysis}

Statistical analyses were performed using Statistical Analysis System (SAS) for OS/2, version 9.3 (SAS Institute, Inc., Cary, NC, USA). Chi-square test, Fisher's exact test, $t$-test, and logistic regression analysis were used to test differences between groups, when appropriate. Statistical significance was defined as $P$-value less than 0.05 .

\section{Results}

A total 491 of patients (644 eyes) were included in this study. The mean duration of follow up was 4.8 years ( \pm 6.8 years). The mean number of ophthalmic visits was eleven $( \pm 14.3)$. Of 491 patients, 153 (31.2\%) had bilateral disease, while $338(68.8 \%)$ had unilateral disease. For those with unilateral disease, the right eye was involved in 178 patients (52.7\%). Of 491 patients, 57 (11.6\%) had acute onset and $158(32.8 \%)$ had insidious disease. A total 276 (56.2\%) patients had chronic uveitis.

\section{Age}

The mean age at presentation was 45.5 years ( \pm 21.3 years) $(\mathrm{n}=491)$, while the mean age at diagnosis was 46.0 years ( \pm 21.4 years) (Figure 2$)$.

Table 1 shows the breakdown of our population into four groups.

\section{Univariate analysis}

Sex

Of 491 patients, 278 (56.6\%) were females, with female: male ratio of 1.3:1.
Table I Age groups

\begin{tabular}{lll}
\hline Age group (years)* & Patients, $\mathbf{n}$ & Percentage \\
\hline$<18$ & 39 & 7.9 \\
$18-35$ & 129 & 26.3 \\
$18-65$ & 355 & 72.3 \\
$>65$ & 97 & 19.8 \\
\hline
\end{tabular}

Notes: $*$ The groups were defined as: $<18$ years = pediatric group; $18-35$ years $=$ young adults; $18-65$ years $=$ working-age group; $>65$ years $=$ older-age group

Diagnoses by sex are given in Table 2 . Common diagnoses among females were undifferentiated anterior uveitis, traumatic uveitis, herpetic disease, and human leukocyte antigen (HLA)-B27-associated uveitis. Among males, frequent causes were undifferentiated anterior, post-procedural (following cataract, glaucoma, and cornea surgery or intravitreal injections), trauma, and HLA-B27-related anterior uveitis.

\section{Ethnicity}

A mixed ethnic distribution was seen with 297 (60.5\%) Caucasians, 134 (27.3\%) African Americans, 48 (9.8\%) "Others" (Asian, Indian American, or unidentified), and 12 (2.4\%) Hispanics.

The leading cause of uveitis among African American patients was undifferentiated anterior uveitis $(29.9 \%)$, followed by sarcoidosis (17.2\%) (Table 3). The most common causes of uveitis among the 297 Caucasian patients were undifferentiated anterior uveitis $(\mathrm{n}=63$ [21.2\%]) and trauma $(\mathrm{n}=35[11.8 \%])$. Sarcoidosis was significantly more common among African Americans than Caucasians $(P<0.001)$, and herpetic anterior uveitis was significantly more common among Caucasians $(P<0.001)$.

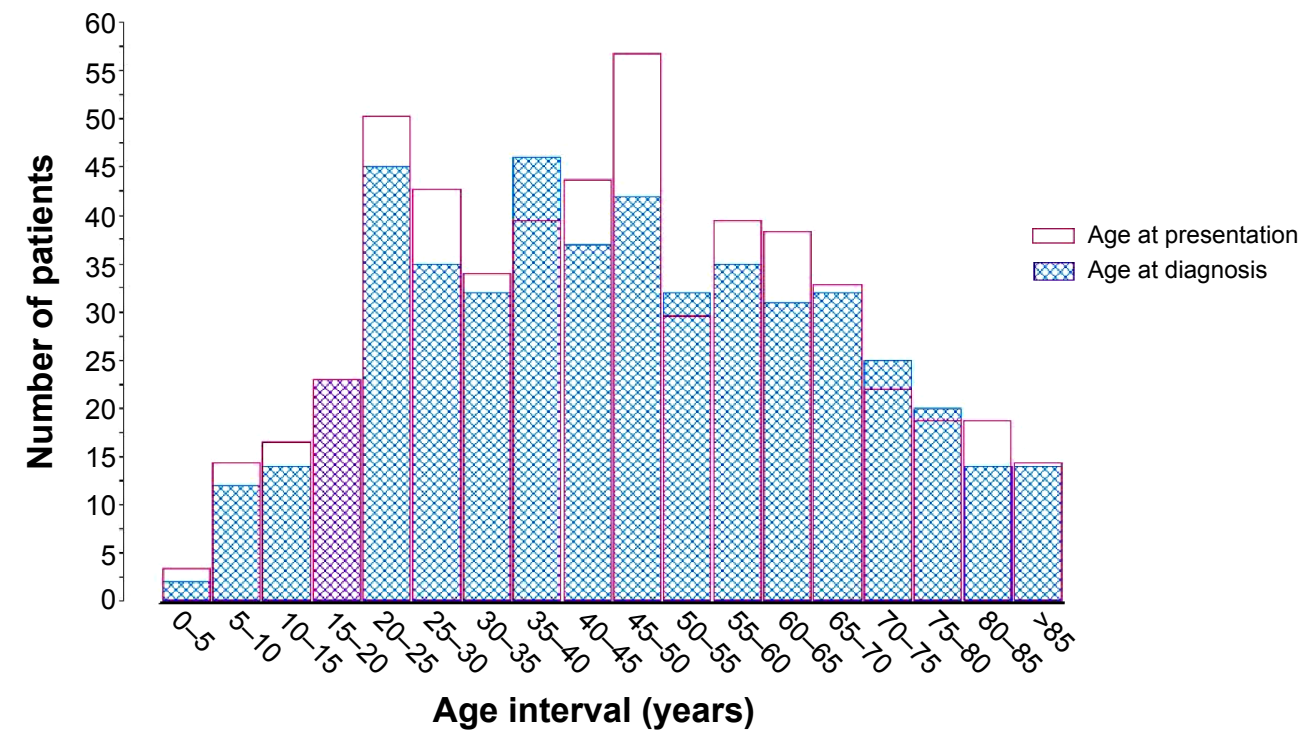

Figure 2 Mean age at diagnosis and presentation.

Note: Intervals are non-overlapping. Each interval is open on the left and closed on the right (eg, [0, 10 years]). 
Table 2 Distribution of diagnoses by sex

\begin{tabular}{|c|c|c|c|}
\hline Diagnosis & $\begin{array}{l}\text { Female } \\
\text { n (\%) }\end{array}$ & $\begin{array}{l}\text { Male } \\
\text { n (\%) }\end{array}$ & $P$-value* \\
\hline Anterior uveitis, undifferentiated & $73(26.3)$ & $53(24.9)$ & 0.755 \\
\hline Trauma & $40(14.3)$ & $20(9.4)$ & 0.098 \\
\hline \multicolumn{4}{|l|}{ Viral } \\
\hline Herpetic anterior uveitis & $30(10.8)$ & $9(4.2)$ & 0.011 \\
\hline Acute retinal necrosis & $9(3.2)$ & $4(1.9)$ & 0.408 \\
\hline CMV uveitis & $2(0.7)$ & $2(0.9)$ & 1.000 \\
\hline Herpetic panuveitis & $\mathrm{I}(0.4)$ & $0(0.0)$ & 1.000 \\
\hline Post-procedural uveitis & $21(7.6)$ & $28(13.1)$ & 0.048 \\
\hline Anterior uveitis HLA-B27 & $18(6.5)$ & $15(7.0)$ & 0.857 \\
\hline Sarcoidosis & $17(6.1)$ & $16(7.5)$ & 0.588 \\
\hline Intermediate uveitis & II (4.0) & $8(3.8)$ & 1.000 \\
\hline Panuveitis, undifferentiated & II (4.0) & $12(5.6)$ & 0.397 \\
\hline Others & $8(2.9)$ & II (5.2) & 0.240 \\
\hline \multicolumn{4}{|l|}{ Infectious, nonviral } \\
\hline Toxoplasma & $8(2.9)$ & $6(2.8)$ & 1.000 \\
\hline Fungal & $3(1.1)$ & $\mathrm{I}(0.5)$ & 0.637 \\
\hline Syphilis & I (0.4) & $0(0.0)$ & 1.000 \\
\hline Tuberculosis & $\mathrm{I}(0.4)$ & $0(0.0)$ & 1.000 \\
\hline Posterior uveitis, undifferentiated & $6(2.2)$ & $3(1.4)$ & 0.738 \\
\hline \multicolumn{4}{|l|}{ White dot syndrome } \\
\hline Birdshot & $5(1.8)$ & $2(0.9)$ & 0.704 \\
\hline MEWDS & $2(0.7)$ & $\mathrm{I}(0.5)$ & 1.000 \\
\hline Multifocal choroiditis & $\mathrm{I}(0.4)$ & $8(3.8)$ & 0.012 \\
\hline APMPPE & $\mathrm{I}(0.4)$ & $3(1.4)$ & 0.321 \\
\hline Serpiginous & I (0.4) & $2(0.9)$ & 0.582 \\
\hline \multicolumn{4}{|l|}{ Seronegative SpA } \\
\hline Ankylosing spondylitis & $2(0.7)$ & $3(1.4)$ & 0.657 \\
\hline Crohn's disease & $2(0.7)$ & $2(0.9)$ & 1.000 \\
\hline Ulcerative colitis & $\mathrm{I}(0.4)$ & $\mathrm{I}(0.5)$ & 1.000 \\
\hline Reactive arthritis & I (0.4) & $0(0.0)$ & 1.000 \\
\hline Psoriatic arthritis & $0(0.0)$ & $\mathrm{I}(0.5)$ & 0.434 \\
\hline Eales disease & I (0.4) & $0(0.0)$ & 1.000 \\
\hline \multicolumn{4}{|l|}{ Collagen vascular disease } \\
\hline Polyarteritis nodosa & $\mathrm{I}(0.4)$ & $0(0.0)$ & 1.000 \\
\hline Granulomatosis with polyangiitis & $0(0.0)$ & $\mathrm{I}(0.5)$ & 0.434 \\
\hline Total & $279(100.0)$ & $214(100.0)$ & $0.066^{\#}$ \\
\hline
\end{tabular}

Notes: *Fisher's exact test on $2 \times 2$ table, eg, female vs male compared on sarcoidosis vs all other diagnoses. "Fisher's exact test applied to entire table, using Monte Carlo sampling $(P=0.085)$.

Abbreviations: APMPPE, acute posterior multifocal placoid pigment epitheliopathy; CMV, cytomegalovirus; HLA, human leukocyte antigen; MEWDS, multiple evanescent white dot syndrome; seronegative SpA, seronegative spondyloarthropathy.

\section{Multivariate analysis}

Multivariate analysis was performed for diagnoses in which a significant difference was found for either race or sex, to assess the contributions of each factor. The data in Table 4 were obtained by fitting separate logistic regression models for each disease. Each model used sex and race as predictor variables.

Herpetic anterior uveitis was more common among Caucasians ( $\mathrm{n}=32[0.8 \%])$ than among African Americans $(\mathrm{n}=2[1.5 \%])$ (sex-adjusted odds ratio: $7.69,95 \%$ confidence interval $[\mathrm{CI}][2.12,50.00])$, while sarcoidosis was more frequent among African Americans ( $\mathrm{n}=23$ [17.2\%]) than
Table 3 Distribution of diagnoses by race

\begin{tabular}{|c|c|c|c|}
\hline Diagnosis & $\begin{array}{l}\text { African } \\
\text { American } \\
\mathbf{n}(\%)\end{array}$ & $\begin{array}{l}\text { Caucasian } \\
\text { n (\%) }\end{array}$ & $P$-value* \\
\hline \multicolumn{4}{|l|}{ Viral } \\
\hline Herpetic anterior uveitis & $2(1.5)$ & $32(10.8)$ & $<0.001$ \\
\hline Acute retinal necrosis & $5(3.7)$ & $8(2.7)$ & 0.553 \\
\hline CMV uveitis & $\mathrm{I}(0.7)$ & $3(1.0)$ & 1.000 \\
\hline Herpetic panuveitis & $0(0.0)$ & $\mathrm{I}(0.3)$ & 1.000 \\
\hline Sarcoidosis & $23(17.2)$ & $9(3.0)$ & $<0.001$ \\
\hline $\begin{array}{l}\text { Anterior uveitis, } \\
\text { undifferentiated }\end{array}$ & $40(29.9)$ & $63(21.2)$ & 0.067 \\
\hline Trauma & $22(16.4)$ & $35(11.8)$ & 0.219 \\
\hline Post-procedural & $14(10.4)$ & $27(9.1)$ & 0.723 \\
\hline Anterior uveitis HLA-B27 & $8(6.0)$ & $22(7.4)$ & 0.686 \\
\hline Others & $2(1.5)$ & $15(5.1)$ & 0.108 \\
\hline Intermediate uveitis & $3(2.2)$ & $14(4.7)$ & 0.291 \\
\hline Panuveitis, undifferentiated & $7(5.2)$ & $13(4.4)$ & 0.805 \\
\hline \multicolumn{4}{|c|}{ Infectious (bacterial, fungal, parasitic) } \\
\hline Toxoplasma & I (0.7) & $10(3.4)$ & 0.185 \\
\hline Fungal & $0(0.0)$ & $4(1.3)$ & 0.315 \\
\hline Syphilis & $0(0.0)$ & $\mathrm{I}(0.3)$ & 1.000 \\
\hline Tuberculosis & $0(0.0)$ & $0(0.0)$ & 1.000 \\
\hline $\begin{array}{l}\text { Posterior uveitis, } \\
\text { undifferentiated }\end{array}$ & $2(1.5)$ & $6(2.0)$ & 1.000 \\
\hline \multicolumn{4}{|l|}{ White dot syndrome } \\
\hline Multifocal choroiditis & $3(2.2)$ & $5(1.7)$ & 0.708 \\
\hline Birdshot & $0(0.0)$ & $5(1.7)$ & 0.330 \\
\hline APMPPE & $0(0.0)$ & $4(1.3)$ & 0.315 \\
\hline Serpiginous & $0(0.0)$ & $3(1.0)$ & 0.556 \\
\hline MEWDS & $0(0.0)$ & $2(0.7)$ & 1.000 \\
\hline \multicolumn{4}{|l|}{ Seronegative SpA } \\
\hline Ankylosing spondylitis & $0(0.0)$ & $5(1.7)$ & 0.330 \\
\hline Crohn's disease & $0(0.0)$ & $4(1.3)$ & 0.315 \\
\hline Reactive arthritis & $0(0.0)$ & $\mathrm{I}(0.3)$ & 1.000 \\
\hline Ulcerative colitis & $0(0.0)$ & $\mathrm{I}(0.3)$ & 1.000 \\
\hline Psoriatic arthritis & $0(0.0)$ & $\mathrm{I}(0.3)$ & 1.000 \\
\hline \multicolumn{4}{|l|}{ Collagen vascular disease } \\
\hline $\begin{array}{l}\text { Granulomatosis with } \\
\text { polyangiitis }\end{array}$ & $0(0.0)$ & $\mathrm{I}(0.3)$ & 1.000 \\
\hline Polyarteritis nodosa & $0(0.0)$ & $\mathrm{I}(0.3)$ & 1.000 \\
\hline Fuch's iridocyclitis & $0(0.0)$ & $\mathrm{I}(0.3)$ & 1.000 \\
\hline Eales disease & $\mathrm{I}(0.8)$ & $0(0.0)$ & 0.311 \\
\hline Total & $134(100.0)$ & $297(100.0)$ & $<0.00 \mathrm{I}^{\dagger}$ \\
\hline
\end{tabular}

Notes: *Fisher's exact test on $2 \times 2$ table, eg, African American vs Caucasian compared on sarcoidosis vs all other diagnoses. 'Fisher's exact test applied to entire table, using Monte Carlo sampling.

Abbreviations: APMPPE, acute posterior multifocal placoid pigment epitheliopathy; CMV, cytomegalovirus; HLA, human leukocyte antigen; MEWDS, multiple evanescent white dot syndrome; seronegative SpA, seronegative spondyloarthropathy.

among Caucasians ( $\mathrm{n}=9$ [3.0\%]) (sex-adjusted odds ratio: $6.54,95 \%$ CI $[2.98,15.29])$. Herpetic anterior uveitis was more common among females ( $\mathrm{n}=30$ [10.8\%]) than among males $(\mathrm{n}=9$ [4.2\%]) (race-adjusted odds ratio: $3.03,95 \% \mathrm{CI}$ $[1.32,7.71])$, while multifocal choroiditis was more common among males $(\mathrm{n}=8[3.8 \%])$ than among females $(\mathrm{n}=1[0.4 \%])$ (race-adjusted odds ratio: 9.09, 95\% CI [1.47, 100.00]). 
Table 4 Multivariate analysis for race and sex

\begin{tabular}{lll}
\hline Diagnosis & \multicolumn{2}{l}{$\begin{array}{l}\text { Odds ratio } \\
\text { (95\% confidence interval)* }\end{array}$} \\
\cline { 2 - 3 } & \multicolumn{1}{l}{ Race } & Sex \\
\hline Sarcoidosis & $6.54[2.98,15.29]$ & $0.89[0.42,1.9 I]$ \\
Anterior uveitis, undifferentiated & $1.58[0.99,2.5 \mathrm{I}]$ & $\mathrm{I} .02[0.65,1.60]$ \\
Herpetic anterior uveitis & $0.13[0.02,0.47]$ & $3.03[1.32,7.7 \mathrm{I}]$ \\
Multifocal choroiditis & $\mathrm{I} .24[0.24,5.52]$ & $0.1 \mathrm{I}[0.01,0.68]$ \\
Trauma & $\mathrm{I} .5 \mathrm{I}[0.83,2.69]$ & $\mathrm{I} .76[0.97,3.25]$ \\
Post-procedural & $\mathrm{I} .14[0.56,2.24]$ & $0.55[0.29,1.06]$ \\
\hline
\end{tabular}

Notes: *Odds ratios were computed via exact logistic regression. The odds ratios are $>$ I when African Americans or females are more likely to have the disease. The odds ratios are statistically significant if the $95 \%$ confidence interval does not include I.

\section{Postal codes}

Of 1,216 postal codes in VA, we covered 137 (11.3\%) (Figure 3). Among the patients studied, 462 patients resided within VA (473 patients resided in the Mid-Atlantic region), and 27 patients were from other states, most commonly from West VA (seven patients) and Maryland (three patients) (Table 5). ${ }^{15}$

\section{Visual acuity and intraocular pressure}

At patient level $(\mathrm{n}=458)$ analysis mean $\log$ MAR visual acuities were $0.54 \pm 0.74$ (Snellen visual acuity 20/70) and $0.52 \pm 0.82$ (Snellen visual acuity 20/66) at baseline and at the end of the study, respectively $(P=0.002)$. At the eye level $(\mathrm{n}=604)$, mean $\log$ MAR was 0.18 (Snellen visual acuity 20/30) at initial and final follow up. Similarly, at patient level mean $\log$ MAR was significantly improved for intermediate uveitis ( $P=0.038)$. The $P$-value for moderate visual loss and severe visual loss was not significant for anterior, posterior, or panuveitis at the end of the study (Table 6). When analyzed at the eye level, no significant difference was seen for any
Table 5 States of residence for patients seeking care at the University of Virginia

\begin{tabular}{ll}
\hline State & Number of patients \\
\hline AL & 1 \\
CA & 1 \\
FL & 2 \\
GA & 2 \\
MD & 3 \\
MI & 2 \\
NC & 1 \\
NM & 1 \\
NY & 1 \\
OR & 1 \\
SC & 2 \\
TN & 1 \\
VA & 462 \\
WA & 1 \\
WV & 7 \\
WY & 1 \\
\hline Ab
\end{tabular}

Abbreviations: AL, Alabama; CA, California; FL, Florida; GA, Georgia; MD, Maryland; MI, Michigan; NC, North Carolina; NM, New Mexico; NY, New York; OR, Oregon; SC, South Carolina; TN, Tennessee; VA, Virginia; WA, Washington; WV, West Virginia; WY, Wyoming.

of the anatomical types of uveitis. Details of visual outcome analysis will follow in a separate paper.

A total of $110(\mathrm{n}=470[17.9 \%])$ of 85 patients presented with ocular hypertension at baseline, while 88 eyes $(n=470$ [16.6\%]) of 74 patients had ocular hypertension at the end of the study. Twenty-six eyes $(n=470[5.3 \%])$ of 25 patients at baseline presented with hypotension, whereas 28 eyes $(n=470$ [6.0\%]) of 26 patients had hypotony at the end of the follow up. Median IOP remained stable with ocular hypertension positively associated with moderate to severe visual loss as compared to normotensive eyes (1.89 times at baseline, 2.62 times at last follow up). Details of IOP outcomes will be included in a separate paper.

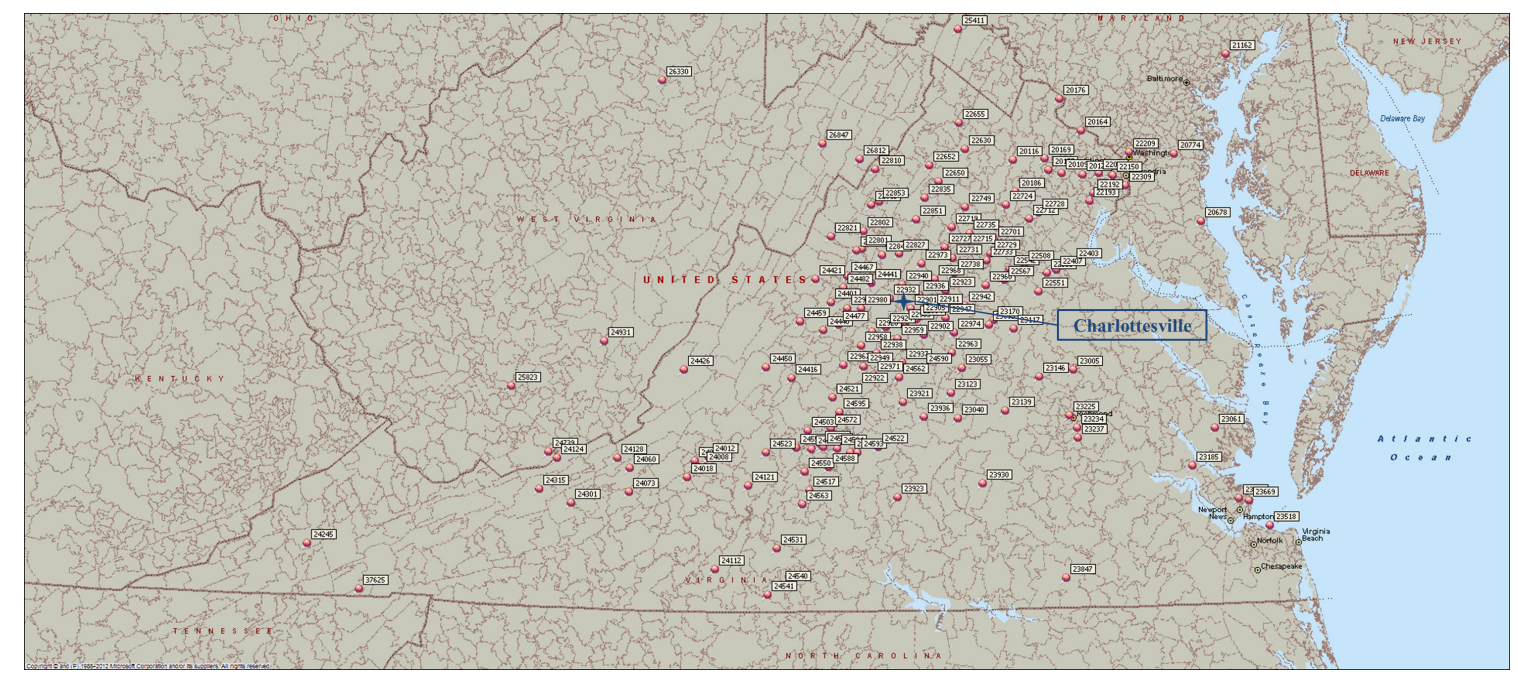

Figure 3 Map of Virginia with the residential postal codes of the patients. 
Table 6 Change in visual acuity

\begin{tabular}{|c|c|c|c|c|c|}
\hline \multicolumn{6}{|l|}{ Overall } \\
\hline Variable & $\mathbf{N}$ & Mean & SD & SE & Median \\
\hline Initial logMAR & 458 & 0.54 & 0.74 & 0.03 & 0.3 \\
\hline Final logMAR & 458 & 0.52 & 0.82 & 0.04 & 0.18 \\
\hline \multicolumn{6}{|l|}{$P=0.002 *$} \\
\hline \multicolumn{6}{|l|}{ Anterior uveitis } \\
\hline Initial logMAR & 308 & 0.44 & 0.63 & 0.04 & 0.18 \\
\hline Final $\log M A R$ & 308 & 0.45 & 0.78 & 0.04 & 0.16 \\
\hline \multicolumn{6}{|l|}{$P=0.058^{*}$} \\
\hline \multicolumn{6}{|c|}{ Intermediate uveitis } \\
\hline Initial logMAR & 25 & 0.61 & 0.83 & 0.17 & 0.3 \\
\hline Final logMAR & 25 & 0.27 & 0.35 & 0.07 & 0.14 \\
\hline \multicolumn{6}{|l|}{$P=0.038^{*}$} \\
\hline \multicolumn{6}{|l|}{ Posterior uveitis } \\
\hline Initial logMAR & 64 & 1.07 & 1.05 & 0.13 & 0.63 \\
\hline Final logMAR & 64 & 0.99 & 1.05 & 0.13 & 0.53 \\
\hline \multicolumn{6}{|l|}{$P=0.197^{*}$} \\
\hline \multicolumn{6}{|l|}{ Panuveitis } \\
\hline Initial logMAR & 57 & 0.43 & 0.4 & 0.05 & 0.39 \\
\hline Final logMAR & 57 & 0.45 & 0.63 & 0.08 & 0.25 \\
\hline$P=0.216^{*}$ & & & & & \\
\hline
\end{tabular}

Note: *Wilcoxon rank sum test, analysis done at patient level.

Abbreviations: SD, standard deviation; SE, standard error of the mean.

\section{Treatment received}

A total of 365 (74.6\%) and 133 (27.3\%) patients received local or systemic steroids, respectively. Antimetabolites were given to $52(10.6 \%)$ patients, while anti-TNF agents were given in $17(3.5 \%)$ cases.

In all, 116 patients $(23.6 \%)$ were managed with topical medication; 43 patients (8.8\%) underwent glaucoma surgery. One hundred and twenty nine (26.4\%) had cataract extraction, and 46 (9.4\%) had pars plana vitrectomy. Table 7 shows treatment received and complications, like glaucoma, cataract, and pars plana vitrectomy.

\section{Anatomical localization}

The distribution of cases by anatomic site of inflammation is given in Table 8. Anterior uveitis was most common, followed by panuveitis and posterior uveitis.

Table 7 Ophthalmic management and interventions

\begin{tabular}{ll}
\hline Treatment/intervention & Number (\%) \\
\hline Local steroids & $365(74.6 \%)$ \\
Systemic steroids & $133(27.3 \%)$ \\
Anti-TNF agents & $17(3.5 \%)$ \\
Antimetabolites & $52(10.6 \%)$ \\
Intravitreal injection & $54(11.0 \%)$ \\
Sub-Tenon injection & $23(4.7 \%)$ \\
Glaucoma topical treatment & $116(23.6 \%)$ \\
Glaucoma surgery & $43(8.8 \%)$ \\
Cataract surgery & $129(26.4 \%)$ \\
Pars plana vitrectomy & $46(9.4 \%)$ \\
\hline
\end{tabular}

Abbreviation: TNF, tumor necrosis factor.
Table 8 Anatomical classification

\begin{tabular}{lllll}
\hline Definition & Number & Percentage & $\begin{array}{l}\text { Number } \\
\text { bilateral }\end{array}$ & $\begin{array}{l}\text { Number } \\
\text { unilateral }\end{array}$ \\
\hline Anterior uveitis & 332 & 67.6 & 75 & 257 \\
Intermediate uveitis & 26 & 5.3 & 19 & 7 \\
Posterior uveitis & 62 & 12.6 & 32 & 30 \\
Panuveitis & 71 & 14.5 & 44 & 27 \\
\hline
\end{tabular}

\section{Differentiated vs undifferentiated}

A total of 335 patients (68.2\%) with uveitis met criteria for a specific etiology (Figure 4). Of these, 63 (18.8\%) patients were diagnosed at first consultation, and the remaining 272 $(81.2 \%)$, were diagnosed on a subsequent visit.

\section{Clinical classification}

Common uveitis diagnoses were undifferentiated anterior uveitis $(n=126[25.7 \%])$, followed by trauma $(n=60$ $[12.2 \%])$, post-procedural $(\mathrm{n}=49[10.0 \%])$, herpetic anterior uveitis ( $\mathrm{n}=39[7.9 \%]$ ), HLA-B27-associated anterior uveitis $(n=33[6.7 \%])$, and sarcoidosis $(n=33$ [6.7\%]). The relative frequencies of key uveitis diagnoses are shown in Table 9.

Post-procedural uveitis $(n=49$ [10\%]) was defined as ocular inflammation following intraocular surgery, laser, or intravitreal injection. Of these, 19 (48.7\%) were diagnosed post-cataract extraction and intraocular lens (IOL) placement, four $(10.3 \%)$ were post-intravitreal injection, six (15.4\%) were post-cornea surgery, three $(7.7 \%)$ were post-laser procedures, six (15.4\%) were post-retina surgery, and one (2.6\%) was following glaucoma surgery.

A total of 77 patients (16\%) had infectious uveitis. The most common infectious etiology was herpetic anterior uveitis $(n=39$ [50.6\%]), followed by toxoplasma uveitis $(\mathrm{n}=14[18 \%])$. The most prevalent viral pathogen was herpes zoster (varicella zoster virus [VZV]) $(n=21$ [27\%]), followed

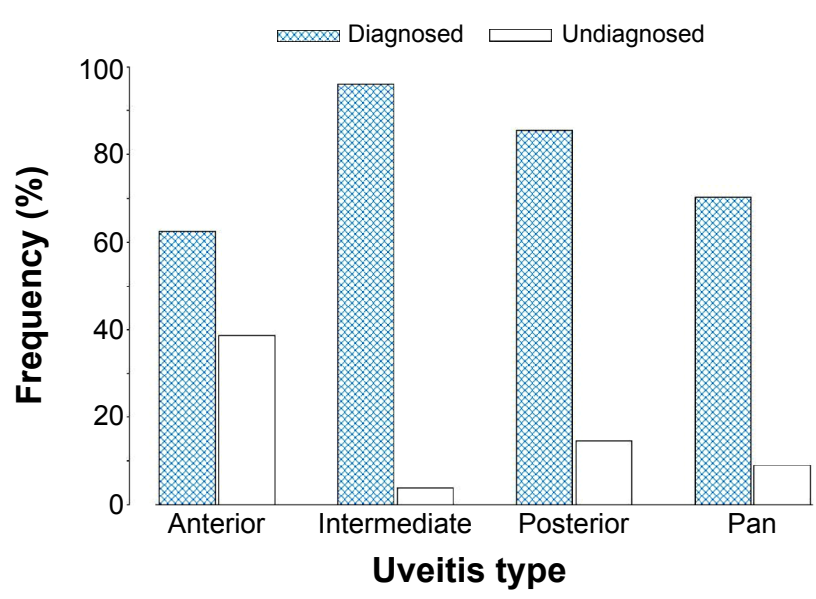

Figure 4 Differentiated (diagnosed) vs undifferentiated (undiagnosed) cases. 
Table 9 Uveitis diagnoses at University of Virginia

\begin{tabular}{|c|c|c|}
\hline Diagnosis & Patients, $n$ & Percentage \\
\hline Anterior uveitis, undifferentiated & 126 & 25.7 \\
\hline Trauma & 60 & 12.2 \\
\hline Post-procedural & 49 & 10.0 \\
\hline \multicolumn{3}{|l|}{ Viral } \\
\hline Acute retinal necrosis & 13 & 2.7 \\
\hline Herpetic anterior uveitis & 39 & 7.9 \\
\hline CMV uveitis & 4 & 0.8 \\
\hline Herpetic panuveitis & I & 0.2 \\
\hline Sarcoidosis & 33 & 6.7 \\
\hline HLA-B27 anterior uveitis & 33 & 6.7 \\
\hline Fuch's iridocyclitis & I & 0.2 \\
\hline Intermediate uveitis & 26 & 5.3 \\
\hline Panuveitis, undifferentiated & 23 & 4.7 \\
\hline \multicolumn{3}{|l|}{ Infectious } \\
\hline Toxoplasma & 14 & 2.9 \\
\hline Syphilis & 1 & 0.2 \\
\hline Tuberculosis & I & 0.2 \\
\hline Fungal & 4 & 0.8 \\
\hline Posterior uveitis, undifferentiated & 9 & 1.8 \\
\hline \multicolumn{3}{|l|}{ White dot syndrome } \\
\hline Multifocal choroiditis & 9 & 1.8 \\
\hline Birdshot & 7 & $\mathrm{I} .4$ \\
\hline APMPPE & 4 & 0.8 \\
\hline Serpiginous & 3 & 0.6 \\
\hline MEWDS & 3 & 0.6 \\
\hline Eales disease & I & 0.2 \\
\hline \multicolumn{3}{|l|}{ Collagen vascular disease } \\
\hline Granulomatosis with polyangiitis & 1 & 0.2 \\
\hline Polyarteritis nodosa & I & 0.2 \\
\hline \multicolumn{3}{|l|}{ Seronegative SpA } \\
\hline Reactive arthritis & I & 0.2 \\
\hline Ankylosing spondylitis & 5 & 1.0 \\
\hline Crohn's disease & 4 & 0.8 \\
\hline Ulcerative colitis & 2 & 0.4 \\
\hline Psoriatic arthritis & I & 0.2 \\
\hline Others & 19 & 3.9 \\
\hline Total & 491 & 100.0 \\
\hline
\end{tabular}

Abbreviations: APMPPE, acute posterior multifocal placoid pigment epitheliopathy; CMV, cytomegalovirus; HLA, human leukocyte antigen; MEWDS, multiple evanescent white dot syndrome; seronegative SpA, seronegative spondyloarthropathy.

by herpes simplex virus (HSV) $(\mathrm{n}=20[26 \%])$. Acute retinal necrosis (ARN) was diagnosed in 14 patients (18\%). Aqueous humor was analyzed in all 14 patients with ARN and was positive in seven patients (50\%). Of the 77 patients with infectious etiology, 4 (4\%) had fungal endophthalmitis, one had syphilitic chorioretinitis (1\%), and one had tuberculous uveitis (1\%).

\section{Comparison with other studies}

Data on uveitis type, distribution, and diagnosis were compared with those from studies in other centers. ${ }^{5-11}$ The anatomic distributions of uveitis are shown in Table 10, and the relative frequencies of key uveitis diagnoses are shown in Table 11.

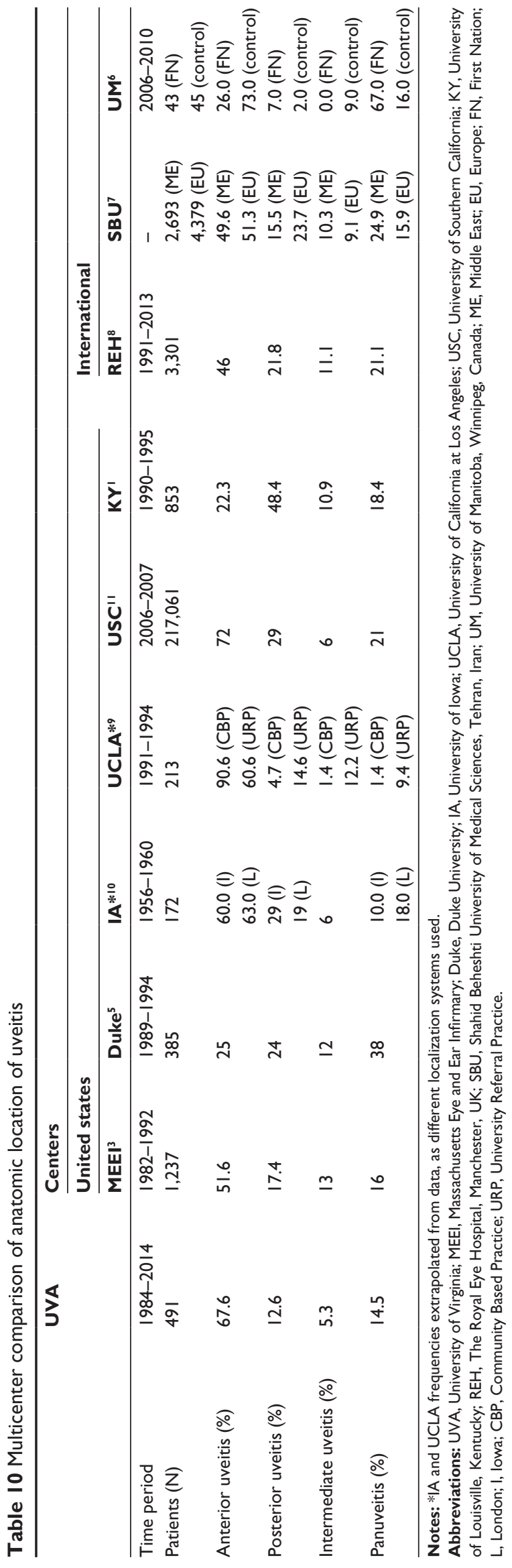


Table I I Relative frequencies of key uveitis diagnoses

\begin{tabular}{|c|c|c|c|c|c|c|c|c|}
\hline & \multirow[t]{4}{*}{ UVA } & \multicolumn{7}{|l|}{ Centers } \\
\hline & & \multicolumn{6}{|c|}{ United States } & \multirow{3}{*}{$\frac{\text { Internationa }}{\text { REH }^{8}}$} \\
\hline & & MEEI $^{3}$ & Duke $^{5}$ & $\mathbf{I A}^{10}$ & UCLA $^{9}$ & USC"I & KY' & \\
\hline & & & & $\begin{array}{l}\text { (IA } \\
\text { frequencies*) }\end{array}$ & $\begin{array}{l}\text { (university } \\
\text { referral } \\
\text { frequencies*) }\end{array}$ & & & \\
\hline Time period & $1984-2014$ & $1982-1992$ & $1989-1994$ & $1956-1960$ & $1991-1994$ & $2006-2007$ & $1990-1995$ & $|99|-2013$ \\
\hline Patients, $\mathrm{n}$ & 491 & $\mathrm{I}, 237$ & 385 & 172 & 213 & 217,061 & 853 & 3,301 \\
\hline Sarcoidosis, \% & 6.7 & 9.6 & II & 5 & 0.8 & 2 & 2.2 & 9.7 \\
\hline Granulomatosis with polyangiitis, \% & 0.2 & & & & & & & \\
\hline Polyarteritis nodosa, \% & 0.2 & 0.2 & & & & & & \\
\hline Anterior uveitis, undifferentiated, \% & 25.7 & 37.8 & 12 & 60 & 30.5 & & 54.2 & 7 \\
\hline Intermediate, \% & 3.9 & & 12 & 6 & 12.2 & & 78.7 & \\
\hline Panuveitis, undifferentiated, \% & 4.7 & 22.2 & 17 & & 40 & & 30.9 & 6.6 \\
\hline Posterior uveitis, undifferentiated, \% & 1.8 & 13.3 & 2 & 17 & & & 19.3 & \\
\hline Toxoplasma, \% & 2.9 & 4.8 & 10 & 9 & & 4 & 3.9 & 6.9 \\
\hline Syphilis, \% & 0.2 & 1.7 & 0.5 & I & & & & \\
\hline Tuberculosis, \% & 0.2 & 0.6 & 0.5 & & & 0.4 & & \\
\hline Fungal, \% & 0.8 & 0.3 & & & & & & \\
\hline Reactive arthritis, \% & 0.2 & & & & & I & & \\
\hline Ankylosing spondylitis, \% & I & 3.8 & 2 & & & 3 & & \\
\hline Crohn's disease, \% & 0.8 & 0.7 & & & & & & \\
\hline Ulcerative colitis, \% & 0.4 & 0.5 & & & & & & \\
\hline Psoriatic arthritis, \% & 0.2 & & & & & & & \\
\hline Acute retinal necrosis, $\%$ & 2.7 & & 2 & & & & & \\
\hline Herpetic anterior uveitis, \% & 7.9 & 9.7 & 3 & & & & & \\
\hline CMV uveitis, \% & 0.8 & 2.2 & & & & & 15.1 & \\
\hline Herpetic panuveitis, \% & 0.2 & & & & & & & \\
\hline Multifocal choroiditis, \% & 1.8 & & 6 & & 6.5 & & 4.9 & \\
\hline Birdshot, \% & 1.4 & & 0.78 & & 6.5 & I & 1.6 & \\
\hline APMPPE, \% & 0.8 & & 0.5 & & & & & \\
\hline Serpiginous, \% & 0.6 & & & & 3.2 & & 1.9 & \\
\hline MEWDS, \% & 0.6 & & & & & 0.4 & & \\
\hline Trauma, \% & 12.2 & & & & & & & \\
\hline Post-procedural, \% & 10 & & & & & & & \\
\hline Fuch's iridocyclitis, \% & 0.2 & & 2 & & & I & & 11.5 \\
\hline Eales disease, \% & 0.2 & & & & & & & \\
\hline Anterior uveitis HLA-B27, \% & 6.7 & 31.3 & 19 & & 17.8 & & 8.4 & 3.6 \\
\hline Others, \% & 3.9 & & & & & & & \\
\hline
\end{tabular}

Notes: *IA and UCLA frequencies extrapolated from data, as different localization systems were used.

Abbreviations: APMPPE, acute posterior multifocal placoid pigment epitheliopathy; CMV, cytomegalovirus; Duke, Duke University; HLA, human leukocyte antigen; IA, University of lowa; KY, University of Louisville, Kentucky; MEEI, Massachusetts Eye and Ear Infirmary; MEWDS, multiple evanescent white dot syndrome; REH, Royal Eye Hospital, Manchester, UK; UCLA, University of California at Los Angeles; USC, University of Southern California; UVA, University of Virginia.

Comparison of anterior, intermediate, and panuveitis with our study is given in Tables $12-15$.

\section{Discussion}

This series reviewed the epidemiologic features of all patients with uveitis seen over a 30-year period at the UVA located in Charlottesville, VA, USA.

Most patients (72.3\%) were of working age (18-65 years), and the mean number of visits per patient was eleven visits per year, suggesting that many patients may have been unable to maintain employment while being actively managed. This is consistent with other reports that the estimated economic impact of visual loss among patients younger than 40 years of age in the United States is more than $\$ 38$ billion, related to medical care, patient support, and loss of quality of life. ${ }^{16}$

The ratio of females: males was $1.3: 1$, which is close to the frequency and sex ratio reported in other studies. ${ }^{1,3,5,8,17-20}$ For comparison, the female population of VA is $50.8 \%$, with a female:male ratio of $1.03: 1 .^{21}$ Female patients were predominantly seen in five common diagnostic categories, none of which reached statistical 
Table 12 Anterior uveitis diagnoses

\begin{tabular}{|c|c|c|c|c|c|c|c|c|}
\hline & $\begin{array}{l}\text { UVA } \\
n=332 \\
n,(\%)\end{array}$ & $\begin{array}{l}\text { Duke }^{5} \\
\text { n=97 } \\
\% *\end{array}$ & $\begin{array}{l}\text { MEEI }^{3} \\
n=637 \\
\% *\end{array}$ & $\begin{array}{l}\text { USC }^{26} \\
n=167 \\
\% *\end{array}$ & $\begin{array}{l}\text { UCLA**9 } \\
\mathrm{n}=129 \\
\% *\end{array}$ & $\begin{array}{l}\mathbf{K Y} \\
\mathrm{n}=190 \\
\% *\end{array}$ & $\begin{array}{l}\text { IA**10 } \\
\mathbf{n}=104 \\
\% *\end{array}$ & $\begin{array}{l}\mathbf{R E H} * * 8 \\
\mathbf{n}=3,301 \\
\% *\end{array}$ \\
\hline Undifferentiated & $126(38)$ & 49 & 37.8 & 12.1 & 50.5 & 54.2 & 60 & 12.3 \\
\hline Trauma & $59(17.8)$ & & & 0.8 & & 2.7 & & \\
\hline Herpetic uveitis & $39(11.7)$ & 3.0 & 9.7 & 9.0 & & 2.2 & & 1.7 \\
\hline Post-procedural & $36(10.5)$ & & & & & & & \\
\hline HLA-B27 & $33(9.9)$ & 19.0 & 6.4 & 3.0 & 17.8 & 8.4 & & 4.5 \\
\hline Sarcoidosis & $19(5.7)$ & 3.0 & 5.8 & & & 1.2 & 11.0 & 2.39 \\
\hline Ankylosing spondylitis & $4(1.2)$ & 9.0 & 7.4 & 1.5 & & 4.7 & 13.0 & \\
\hline IBD & $4(1.2)$ & 1.0 & 2.4 & 0.7 & & & 7.0 & 0.96 \\
\hline Reactive & $\mathrm{I}(0.3)$ & 3.0 & 4.0 & 1.0 & & & 12.0 & 0.66 \\
\hline Psoriatic arthritis & $\mathrm{I}(0.3)$ & & 0.8 & & & & & 0.57 \\
\hline Fuch's heterochromic iridocyclitis & $\mathrm{I}(0.3)$ & 7.0 & 5.0 & 1.8 & & 2.2 & 39.0 & 11.5 \\
\hline Granulomatosis with polyangiitis & $\mathrm{I}(0.3)$ & & 0.5 & & & & & \\
\hline Others & $8(2.7)$ & & & & & & & \\
\hline
\end{tabular}

Notes: Others included: TINU (3), rheumatoid arthritis (I), juvenile rheumatoid arthritis (I), multiple sclerosis (I), and Posner-Schlossman syndrome (2). *All values given as percentage only. **UCLA, IA, and REH values extrapolated from given data.

Abbreviations: Duke, Duke University; HLA, human leukocyte antigen; IA, University of lowa; IBD, Inflammatory bowel disease; KY, University of Louisville, Kentucky; MEEI, Massachusetts Eye and Ear Infirmary; REH, Royal Eye Hospital, Manchester, UK; TINU, tubulointerstitial nephritis and uveitis; UCLA, University of California at Los Angeles; USC, University of Southern California; UVA, University of Virginia.

significance on univariate analysis: undifferentiated anterior uveitis, traumatic uveitis, herpetic anterior uveitis, post-procedural, and HLA-B27-associated anterior uveitis. The cause of these types of uveitis being more common in females is not known.

Our study had a higher proportion of African American patients $(\mathrm{n}=134[27 \%])$ than reported in most other studies (10\% in Amsterdam, $13.7 \%$ at the University of Louisville, Kentucky, 5.6\% in at the University of California, Los Angeles [UCLA], and $5.8 \%$ at the Massachusetts Eye and Ear Infirmary). ${ }^{1,5,9,28}$ Merrill et $\mathrm{al}^{5}$ reported an African American population of 120 of $385(31 \%)$ at Duke University in the neighboring state of NC. For comparison, Charlottesville, VA has a $69.1 \%$ Caucasian and $19.4 \%$ African American population. Duke is situated in an area with $42.5 \%$ Caucasians and $41.0 \%$ African Americans. ${ }^{21}$ The percentage of patients with sarcoidosis varies based on region: studies report $5 \%$ or less in Portugal, Portland, University of South California, and Iowa; 9\% in Amsterdam, and 11\% at Duke. ${ }^{5,10,22-25}$ According to Merrill et al the frequencies of sarcoidosis were $25 \%$ for African American (30/120) and 5\% (14/265) for all
non-African American patients. ${ }^{5}$ At UVA, the incidence was $17.2 \%$ for African American (23/134) and 3\% (9/297) for Caucasian patients.

We compared race and sex data for each entity with the race or sex distributions of our uveitis population as a whole. Multivariate analysis revealed significant differences between races for sarcoidosis and herpetic anterior uveitis, as well as significant sex differences for multifocal choroiditis and herpetic anterior uveitis. Herpetic anterior uveitis was significantly more prevalent among Caucasian females and multifocal choroiditis among males. Also, sarcoidosis was significantly more common among African Americans than Caucasians. Factors that influence the occurrence of sarcoidosis manifestations may be familial, genetic, and environmental..$^{39}$ Cozier et al reported a higher incidence of sarcoidosis in young African American women. A cohort of 59,000 female participants (1995-2007) between the ages of 21-69 years was surveyed in regards to their health in 13 US states and the District of Colombia. Sarcoidosis can be inherited if either a first- or second-degree relative is affected by the condition. ${ }^{40}$

Table 13 Intermediate uveitis diagnoses

\begin{tabular}{|c|c|c|c|c|c|c|c|c|}
\hline & $\begin{array}{l}\text { UVA } \\
n=26 \text { (\%) }\end{array}$ & $\begin{array}{l}\text { Duke }^{5} \\
\mathrm{n}=47^{*}\end{array}$ & $\begin{array}{l}M^{M E E I^{3}} \\
n=162^{*}\end{array}$ & $\begin{array}{l}\text { USC }^{26} \\
\mathrm{n}=92^{*}\end{array}$ & $\begin{array}{l}\text { UCLA**9 } \\
\mathrm{n}=\mathbf{2 6 *}\end{array}$ & $\begin{array}{l}K Y^{\prime} \\
n=94\end{array}$ & $\begin{array}{l}\mid A * * 10 \\
\mathbf{n}=52 *\end{array}$ & $\begin{array}{l}\text { REH } * * 8 \\
\mathbf{n}=3,30 \text { I* }\end{array}$ \\
\hline Undifferentiated & $20(77)$ & 98 & 69.1 & 100 & 30.8 & 94.7 & 40.0 & 7.8 \\
\hline Sarcoidosis & $3(11.5)$ & 2.0 & 22.2 & & 0 & & & 1.75 \\
\hline Multiple sclerosis & $2(3.8)$ & & 8.0 & & 0 & 2.1 & & 0.54 \\
\hline Ankylosing spondylitis & I (3.8) & & & & & & & \\
\hline
\end{tabular}

Notes: *All values given as percentage only. **UCLA, IA, and REH values extrapolated from given data.

Abbreviations: Duke, Duke University; IA, University of lowa; KY, University of Louisville, Kentucky; MEEl, Massachusetts Eye and Ear Infirmary; REH, Royal Eye Hospital, Manchester, UK; UCLA, University of California at Los Angeles; USC, University of Southern California; UVA, University of Virginia. 
Table I 4 Posterior uveitis diagnoses

\begin{tabular}{|c|c|c|c|c|c|c|c|c|}
\hline & $\begin{array}{l}\text { UVA } \\
n=62(\%)\end{array}$ & $\begin{array}{l}\text { Duke }^{5} \\
\mathrm{n}=93 *\end{array}$ & $\begin{array}{l}\text { MEEI }^{3} \\
n=240 *\end{array}$ & $\begin{array}{l}\text { USC }^{26} \\
n=230 *\end{array}$ & $\begin{array}{l}\text { UCLA**9 } \\
\mathbf{n = 3} \text { I* }\end{array}$ & $\begin{array}{l}K Y^{\prime} \\
n=4 \mid 4 *\end{array}$ & $\begin{array}{l}\text { IA**10 } \\
\mathbf{n}=52 *\end{array}$ & $\begin{array}{l}\mathbf{R E H} * * 8 \\
\mathbf{n}=3,301 *\end{array}$ \\
\hline Toxoplasma & II (I7.7) & 42.0 & 24.6 & 9.0 & 35.5 & 7.7 & 65.0 & 6.9 \\
\hline Multifocal choroiditis & $9(14.5)$ & & & 18.0 & 9.7 & 10.1 & & 0.8 \\
\hline Undifferentiated & $9(14.5)$ & 9 & 13.3 & 15.0 & 16.2 & 19.3 & 48.0 & \\
\hline Acute retinal necrosis & $4(6.5)$ & 9 & 5.5 & & & 2.7 & & 0.2 \\
\hline Birdshot & 7 (I I.3) & 3 & 7.9 & 3.0 & 6.5 & 3.4 & & 1.09 \\
\hline MEWDS & $3(4.8)$ & & 1.25 & & & 1.2 & & 0.4 \\
\hline APMPPE & $3(4.8)$ & 2 & 2.0 & & & & & \\
\hline Serpiginous & $3(4.8)$ & & 1.6 & 5.0 & 3.2 & 3.9 & & 0.3 \\
\hline Sarcoidosis & $2(3.2)$ & & 7.5 & 3.9 & & 2.2 & 4.0 & 2.9 \\
\hline CMV & $2(3.2)$ & & 11.6 & 7.0 & & 31.2 & & 0.7 \\
\hline Syphilis & I (I.6) & & 2.0 & & & & 4.0 & \\
\hline Polyarteritis nodosa & I (I.6) & & 0.83 & & & & & \\
\hline Eales disease & I (I.6) & & & & & & & \\
\hline Others & $2(3.2)$ & & & & & & & \\
\hline
\end{tabular}

Notes: Others included: IRVAN (2). *All values given as percentage only. **UCLA, IA, and REH values extrapolated from given data.

Abbreviations: APMPPE, acute posterior multifocal placoid pigment epitheliopathy; CMV, cytomegalovirus; Duke, Duke University; IA, University of lowa; IRVAN, idiopathic retinitis, vasculitis, aneurysms, and neuroretinitis; KY, University of Louisville, Kentucky; MEEl, Massachusetts Eye and Ear Infirmary; MEWDS, multiple evanescent white dot syndrome; REH, Royal Eye Hospital, Manchester, UK; USC, University of Southern California; UCLA, University of California at Los Angeles; UVA, University of Virginia.

In a population-based survey in Rochester, $\mathrm{MN}$, conducted over a 10 -year period, Darrell et al found that the prevalence for all types of uveitis was 204 cases per 100,000 population. They found $54.1 \%$ of all uveitis cases to be anterior, $31.1 \%$ to be posterior, and $14.8 \%$ to be panuveitis. The annual incidence was 17 new cases per 100,000 population; anterior uveitis occurred four times as frequently as posterior uveitis. ${ }^{26} \mathrm{McCannel}$ et al reported that most uveitis case series have been reported from tertiary care centers and, therefore, may be affected by referral bias. ${ }^{9}$

Most (32.1\%) of our patients had a chronic onset of uveitis. This may be due to referral bias, as acute uveitis was more likely to have been treated by local ophthalmologists and not referred to tertiary care centers. ${ }^{3}$ The frequencies for various forms of uveitis in our study were similar to those of many previous studies; for example, reported rates of anterior uveitis in previous studies ranged from $27.8 \%$ to $72 \% .^{11,25-28}$ Some university centers with lower rates of anterior uveitis $(22.3 \%-27.8 \%)$ and a higher rate of posterior uveitis $(38.4 \%-48.4 \%)$ or panuveitis $(38 \%)$ than in our study or in other reports suggested that the discrepancy was due to inclusion of all uveitis cases from their retina service. . $^{1,5,24}$

In our study, the proportion of patients with uveitis for whom a diagnosis could be made was $68.2 \%$. This is higher than rates of diagnosis published by other university referral practice-based investigators, which range from $46 \%$ to $67 \%{ }^{3,23,25-28}$ This could be explained by better diagnostic techniques over the latter period of our study. Also, definitive diagnosis was often made during long-term follow up

Table 15 Panuveitis diagnoses

\begin{tabular}{|c|c|c|c|c|c|c|c|c|}
\hline & $\begin{array}{l}\text { UVA } \\
n=7 \mid \text { (\%) }\end{array}$ & $\begin{array}{l}\text { Duke }^{5} \\
n=148^{*}\end{array}$ & $\begin{array}{l}\text { MEEI }^{3} \\
\mathrm{n}=198^{*}\end{array}$ & $\begin{array}{l}\text { USC }^{26} \\
\mathbf{n}=\mid I^{*}\end{array}$ & $\begin{array}{l}\text { UCLA**9 } \\
\mathbf{n}=\mathbf{2 0} *\end{array}$ & $\begin{array}{l}K Y^{\prime} \\
n=155^{*}\end{array}$ & $\begin{array}{l}\text { IA**10 } \\
\mathrm{n}=18 *\end{array}$ & $\begin{array}{l}\text { REH } * * 8 \\
\mathbf{n}=3,301 *\end{array}$ \\
\hline Undifferentiated & $23(32.4)$ & 43.0 & 22.2 & 45.0 & 18.7 & 30.9 & 27.0 & \\
\hline Post-procedural & $13(18.3)$ & & & & & & & \\
\hline Acute retinal necrosis & $9(12.7)$ & & & 18.0 & & 1.3 & & \\
\hline Sarcoidosis & $9(12.7)$ & 27.0 & 14.1 & 21.0 & 2.3 & 4.5 & 28.0 & 2.99 \\
\hline Toxoplasma & $3(4.2)$ & & & & & & & \\
\hline Fungal & $3(4.2)$ & & 2.0 & & & & & \\
\hline Herpetic uveitis & $2(2.8)$ & & & & & & & 0.01 \\
\hline Trauma & I (I.4) & & & & & & & \\
\hline Tuberculosis & I (I.4) & 1.0 & 2.0 & & & & & \\
\hline Others & $4(5.6)$ & & & & & & & \\
\hline
\end{tabular}

Notes: Others included: Behcet's disease (I), Vogt-Koyanagi-Harada disease (I), juvenile rheumatoid arthritis (I). *All values given as percentage only. **UCLA, IA, and REH values extrapolated from given data.

Abbreviations: Duke, Duke University; IA, University of lowa; KY, University of Louisville, Kentucky; MEEI, Massachusetts Eye and Ear Infirmary; REH, Royal Eye Hospital, Manchester, UK; UCLA, University of California at Los Angeles; USC, University of Southern California; UVA, University of Virginia. 
rather than at the initial visit, which has been emphasized by a previous study. ${ }^{3}$

Comparisons with surveys undertaken in uveitis clinics elsewhere in the world often present great contrasts in the numbers of patients affected within diagnostic categories. ${ }^{3,5,16,18,23,29-37}$ With a combination of different uveitis classification criteria, the most common uveitis diagnoses in our analysis were undifferentiated anterior uveitis (25.4\%), trauma (12.2\%), post-procedural (9.9\%), herpetic anterior uveitis (7.9\%), HLA-B27 anterior uveitis (6.7\%), and sarcoidosis $(6.7 \%)$.

For undifferentiated anterior uveitis, Perkins and Folk investigated the influence of geographic factors on uveitis through the examination of two populations, in London and Iowa (IA). The London population consisted of an urban community, whereas the IA study involved a rural region. They showed no correlation between geographic factors and the type of uveitis manifested. However, the results suggest that genetics has a stronger association than geographic factors. ${ }^{10}$

The most common type of infectious uveitis seen over the study period was herpetic anterior uveitis secondary to VZV or HSV, which is comparable with findings of other American epidemiologic studies. ${ }^{3,11}$ Ocular toxoplasmosis and ARN were also common causes of infectious uveitis.

Table 12 shows the types of anterior uveitis seen in our study population in comparison with other studies. . $^{1,3,5,8-10,11,25}$ We had a large population of patients with traumatic uveitis, which could be relevant to the subset of student population and outdoor sports activities at the UVA. Macewen reported that increasing time available for leisure activities has been parallel to an increase in sport-associated eye trauma. ${ }^{45}$ Traumatic uveitis has been mentioned in only few of the previous studies. The Manchester study included two cases of traumatic anterior uveitis, whereas a UCLA study had $4.8 \%$ of their community-based cases in this category. ${ }^{8,9}$ It has been postulated that the rate at which traumatic anterior uveitis was reported may be artificially high, as trauma may bring patients with preexistent uveitis to the eye clinics. ${ }^{9}$ Also, patients who report to the emergency room at our hospital are commonly evaluated in the eye clinic as well. In contrast to other studies, we classified all uveitis following ophthalmic surgery, intravitreal injection, or laser procedure as post-procedural uveitis. ${ }^{5,8,9}$

Intermediate uveitis is most often undifferentiated. ${ }^{41}$ However, specific systemic syndromes have also been associated with this form of inflammatory eye disease. ${ }^{42}$ In our study, the most common systemic disease association was sarcoidosis, as was reported by Rodriguez et al,
Merrill et al, Henderly et al and also in studies conducted in Rotterdam $(16 \%[n=68])$ and Amsterdam $(9 \%[n=76])^{3,5,22,25,28}$ (Table 11).

Our study and others support toxoplasmosis as the most common cause of posterior uveitis. ${ }^{3,10,25,43}$ Multifocal choroiditis and undifferentiated uveitis were the next common entities in our study. Retinal vasculitis was mentioned as a causative group in previous epidemiologic studies. ${ }^{5,38,44}$ In our patients, retinal vasculitis, a descriptive diagnosis, was seen in many patients with sarcoidosis, Behcet's, Birdshot chorioretinopathy, and Eales disease.

These differences may reflect changes in etiologies over time, as well as better understanding of different uveitic entities, evolution of better diagnostic techniques, and, maybe, real change in disease frequency. More frequent use of PCR on aqueous and vitreous samples, in conjunction with the latest diagnostic tools of ultrawide field imaging and highdefinition OCT permit improved characterization of uveitis and may thereby diminish the number of undifferentiated cases of posterior and panuveitis.

\section{Limitations}

Our uveitis epidemiologic report is retrospective in nature and was largely based on patients referred to our tertiary care center, and therefore, referral bias may exist. Beside true geographic, genetic, environmental, and ethnic differences, other factors may influence the diagnostic variability between different epidemiological studies. Differences in the diagnostic approach of ophthalmologists, availability of uveitis-trained faculty, accessibility to newer diagnostic techniques, and the evolution of diagnostic criteria employed by individual authors are among such factors. Nevertheless, reporting the frequency of various diseases in our area provides a more complete understanding of uveitis in the United States and may help inform future studies.

\section{Conclusion}

In summary, we studied a well-defined population of 491 patients at the UVA Eye Center. To our knowledge, this study of uveitis demographic analysis is unique, as it is the first to be conducted in central Virginia. Over the period of this study, Caucasian patients were more frequently seen than non-Caucasians, but African Americans constituted a considerable size of study population. The frequencies of anterior, intermediate, posterior, and panuveitis were within the range reported by other university-based reports. However, unlike other studies, we had more cases of traumatic and post-procedural uveitis. Common uveitis types observed 
were undifferentiated anterior, traumatic, post-procedural, herpetic disease, HLA-B27 disease, and sarcoidosis. Importantly, mean overall visual acuity remained stable for the cohort from initial to final visit, with the majority receiving topical corticosteroids, confirming that therapeutic interventions are effective.

\section{Acknowledgments}

The authors would like to thank Chang Sup Lee, Vandan Patel, and Shuo Qiu for their contribution in data collection.

A part of this study has been accepted an Association for Research in Vision and Ophthalmology (ARVO) 2015 conference poster.

\section{Disclosure}

The authors report no conflicts of interest in this work.

\section{References}

1. Oruc S, Kaplan AD, Galen M, Kaplan HJ. Uveitis referral pattern in a Midwest University Eye Center. Ocul Immunol Inflamm. 2003;11(4): 287-298.

2. Nussenblatt RB. The natural history of uveitis. Int Ophthalmol. 1990; 14(5-6):303-308.

3. Rodriguez A, Calonge M, Pedroza-Seres M, et al. Referral patterns of uveitis in a tertiary eye care center. Arch Ophthalmol. 1996;114(5): 593-599.

4. Moorthy RS, Rao PK, Read RW, et al. 2011-2012 Basic and Clinical Science Course, Section 9; Intraocular inflammation and uveitis. San Francisco, CA: American Academy of Ophthalmology; 2011.

5. Merrill PT, Kim J, Cox TA, Betor CC, McCallum RM, Jaffe GJ. Uveitis in the southeastern United States. Curr Eye Res. 1997;16(9):865-874.

6. Roy M. Analysis of uveitis in a Canadian aboriginal population. Can J Ophthalmol. 2014;49(2):128-134.

7. Nashtaei EM, Soheilian M, Herbort CP, Yaseri M. Patterns of uveitis in the Middle East and Europe. J Ophthalmic Vis Res. 2011;6(4):233-240.

8. Jones NP. The Manchester Uveitis Clinic: The first 3,000 patientsepidemiology and casemix. Ocul Immunol Inflamm. Epub 2013 Dec 2.

9. McCannel CA, Holland GN, Helm CJ, Cornell PJ, Winston JV, Rimmer TG. Causes of uveitis in the general practice of ophthalmology. UCLA Community-Based Uveitis Study Group. Am J Ophthalmol. 1996;121(1):35-46.

10. Perkins ES, Folk J. Uveitis in London and Iowa. Ophthalmologica. 1984;189(1-2):36-40.

11. Acharya NR, Tham VM, Esterberg E, et al. Incidence and prevalence of uveitis: results from the Pacific Ocular Inflammation Study. JAMA Ophthalmol. 2013;131(11):1405-1412.

12. Yeh S, Forooghian F, Suhler EB. Implications of the Pacific Ocular Inflammation uveitis epidemiology study. JAMA. 2014;311(18): 1912-1913.

13. Bloch-Michel E, Nussenblatt RB. International Uveitis Study Group recommendations for the evaluation of intraocular inflammatory disease. Am J Ophthalmol. 1987;103(2):234-235.

14. Jabs DA, Nussenblatt RB; Rosenbaum JT; The Standardization of Uveitis Nomenclature (SUN) Working Group. Standardization of uveitis nomenclature for reporting clinical data. Results of the First International Workshop. Am J Ophthalmol. 2005;140(3):509-516.

15. usps.com [homepage on the Internet]. Look up a zip code ${ }^{\mathrm{TM}}$. US Postal Service; 2015 [cited November 20, 2014]. Available from: https://tools. usps.com/go/ZipLookupAction!input.action. Accessed February 28, 2015 .
16. Wittenborn JS, Zhang X, Feagan CW, et al; Vision Cost-Effectiveness Study Group. The economic burden of vision loss and eye disorders among the United States population younger than 40 years. Ophthalmology. 2013;120(9):1728-1735.

17. Gritz DC, Wong IG. Incidence and prevalence of uveitis in Northern California; the Northern California Epidemiology of Uveitis Study. Ophthalmology. 2004;111(3):491-500; discussion 500.

18. Singh R, Gupta V, Gupta A. Pattern of uveitis in a referral eye clinic in north India. Indian J Ophthalmol. 2004;52(2):121-125.

19. Khairallah M, Yahia SB, Ladjimi A, et al. Pattern of uveitis in a referral center in Tunisia, North Africa. Eye (Lond). 2007;21(1)33-39.

20. Hamade IH, Elkum N, Tabbara KF. Causes of uveitis at a referral center in Saudi Arabia. Ocul Immunol Inflamm. 2009;17(1):11-16.

21. Census.gov [homepage on the Internet]. State and county QuickFacts. US Census Bureau; 2015 [updated February 5, 2015; cited December 19, 2014]. Available from: http://quickfacts.census.gov/qfd/states/00000. html. Accessed February 28, 2015.

22. Rothova A, Buitenhuis HJ, Meenken C, et al. Uveitis and systemic disease. Br J Ophthalmol. 1992;76(3):137-141.

23. Palmares J, Coutinho MF, Castro-Correia J. Uveitis in northern Portugal. Curr Eye Res. 1990;9 Suppl:S31-S34.

24. Rosenbaum JT. Uveitis. An internist's view. Arch Intern Med. 1989; 149(5):1173-1176.

25. Henderly DE, Genstler AJ, Smith RE, Rao NA. Changing patterns of uveitis. Am J Ophthalmol. 1987;103(2):131-136.

26. Darrell RW, Wagner HP, Kurland LT. Epidemiology of uveitis: incidence and prevalence in a small urban community. Arch Ophthalmol. 1962;68:502-514.

27. James DG, Friedmann AI, Graham E. Uveitis. A series of 368 patients. Trans Ophthalmol Soc UK. 1976;96(1):108-112.

28. Smit RL, Baarsma GS, de Vries J. Classification of 750 consecutive uveitis patients in the Rotterdam Eye Hospital. Int Ophthalmol. 1993; 17(2):71-76.

29. Mercanti A, Parolini B, Bonora A, Lequaglie Q, Tomazzoli L. Epidemiology of endogenous uveitis in north-eastern Italy. Analysis of 655 new cases. Acta Ophthalmol Scand. 2001;79(1):64-68.

30. Tran VT, Auer C, Guex-Crosier Y, Pittet N, Herbort CP. Epidemiology of uveitis in Switzerland. Ocul Immunol Inflamm. 1994;2(3):169-176.

31. Barisani-Asenbauer T, Maca SM, Mejdoubi L, Emminger W, Machold K, Auer H. Uveitis-a rare disease often associated with systemic diseases and infections-a systematic review of 2,619 patients. Orphanet J Rare Dis. 2012;7:57.

32. Yang $\mathrm{P}$, Zhang Z, Zhou H, et al. Clinical patterns and characteristics of uveitis in a tertiary center for uveitis in China. Curr Eye Res. 2005; 30(11):943-948.

33. Soheilian M, Heidari K, Yazdani S, Shahsavari M, Ahmadieh H, Dehghan M. Patterns of uveitis in a tertiary eye care center in Iran. Ocul Immunol Inflamm. 2004;12(4):297-310.

34. Kazokoglu H, Onal S, Tugal-Tutkun I, et al. Demographic and clinical features of uveitis in tertiary centers in Turkey. Ophthalmic Epidemiol. 2008;15(5):285-293.

35. Sittivarakul W, Bhurayanontachai P, Ratanasukon M. Pattern of uveitis in a university-based referral center in southern Thailand. Ocul Immunol Inflamm. 2013;21(1):53-60.

36. Goto H, Mochizuki M, Yamaki K, Kotake S, Usui M, Ohno S. Epidemiological survey of intraocular inflammation in Japan. Jpn J Ophthalmol. 2007;51(1):41-44.

37. Ohguro N, Sonoda KH, Takeuchi M, Matsumura M, Mochizuki M. The 2009 prospective multi-center epidemiologic survey of uveitis in Japan. Jpn J Ophthalmol. 2012;56(5):432-435.

38. Baarsma GS. The epidemiology and genetics of endogenous uveitis: a review. Curr Eye Res. 1992;11 Suppl:S1-S9.

39. Rybicki BA, Iannuzzi MC. Epidemiology of sarcoidosis: recent advances and future prospects. Semin Respir Crit Care Med. 2007;28(1): 22-35.

40. Cozier YC, Berman JS, Palmer JR, Boggs DA, Serlin DM, Rosenberg L. Sarcoidosis in black women in the United States: data from the Black Women's Health Study. Chest. 2011;139(1):144-150. 
41. Aaberg TM. The enigma of pars planitis. Am JOphthalmol. 1987;103(6): $828-830$.

42. LANDERS PH. Vitreous lesions observed in Boeck's sarcoid. Am J Ophthalmol. 1949;32(12):1740.

43. James DG, Friedmann AI, Graham E. Uveitis. A series of 368 patients. Trans Ophthalmol Soc U K. 1976;96(1):108-112.
44. Weiner A, BenEzra D. Clinical patterns and associated conditions in chronic uveitis. Am J Ophthalmol. 1991;112(2):151-158.

45. MacEwen CJ. Sport associated eye injury: a casualty department survey. Br J Ophthalmol. 1987;71(9):701-705.

Clinical Ophthalmology

\section{Publish your work in this journal}

Clinical Ophthalmology is an international, peer-reviewed journal covering all subspecialties within ophthalmology. Key topics include: Optometry; Visual science; Pharmacology and drug therapy in eye diseases; Basic Sciences; Primary and Secondary eye care; Patient Safety and Quality of Care Improvements. This journal is indexed on

Submit your manuscript here: http://www.dovepress.com/clinical-ophthalmology-journal
Dovepress

PubMed Central and CAS, and is the official journal of The Society of Clinical Ophthalmology (SCO). The manuscript management system is completely online and includes a very quick and fair peer-review system, which is all easy to use. Visit http://www.dovepress.com/ testimonials.php to read real quotes from published authors. 